\title{
Theoretical study of lumichrome, 1-methyl-lumichrome and lumiflavin binding ability with thymine
}

\author{
Denisa Cagardová, Martin Michalík, Vladimír Lukeš \\ Institute of Physical Chemistry and Chemical Physics, Slovak University of Technology in Bratislava, \\ Radlinského 9, SK-812 37 Bratislava, Slovakia \\ denisa.cagardova@stuba.sk
}

\begin{abstract}
Gas-phase geometry and electronic structure of lumichrome, 1-methyl-lumichrome and lumiflavin in the electronic ground state and their excited states were investigated using the Density Functional Theory. Their binding ability with thymine was estimated for model van der Waals dimers with two intermolecular hydrogen bonds. The influence of hydrogen bonds on their photophysical properties was analyzed. Obtained theoretical data were compared with available experimental absorption and fluorescence spectra.
\end{abstract}

Keywords: Chemosensor; flavins; fluorescence; hydrogen bond; vertical excited states

\section{Introduction}

Chemosensors are organic or inorganic molecules used for analyte sensing resulting in a detectable change or signal generation (Wu et al., 2017). In this context, changes in the ultraviolet and visible absorption or emission properties are evaluated. Although the fluorescence signal detection is technically more demanding, a low concentration of analytes can be observed with standard fluorescence spectrometers offering the advantage of using the sensors directly within fiber optic systems. Chemosensors can also be applied together with electrochemical methods. All these outlined techniques are suitable for monitoring of various environmental samples or determination of drug concentrations and blood content.

Chemosensors applicable in the recognition of nucleobases have attracted immense interest because of their application in DNA-binding drugs research or in medical analytical chemistry and molecular biology (White et al., 1998). One alternative approach used in the development of these chemical probes involves the use of small molecules (ligands)<smiles>[R7]n1c(=O)[nH]c(=O)c2nc3cc(C)c(C)cc3nc21</smiles>

A/B

Molecule A (Lumichrome): $\mathrm{R}_{1}=\mathrm{H}$ Molecule B (1-Methyl-lumichrome): $\mathrm{R}_{1}=\mathrm{CH}_{3}$ that are able to bind to intrahelical target bases by hydrogen bonds in DNA duplexes (Rajendran and Nair, 2006). Selective binding of these ligand molecules is promoted by a pseudo-base pairing along the nucleobase (Hagihara et al., 2004). From the thermodynamic point of view, pairing of ligands with a nucleon base target can lead to more stable structures than the Watson-Crick base pairing (Lee and Kool, 2005; Kim and Kool, 2005; Gao et al., 2005). Considering chemosensors, benzo[g]pteridine based molecules represent a perspective group. Rajendar et al. (2010) showed that lumichrome (7,8-dimethylbenzo[g]pteridine$2,4(1 \mathrm{H}, 3 \mathrm{H})$-dione) and 1-methyl-lumichrome (1,7,8-trimethylbenzo[g]pteridine-2,4(3H)-dione) selectively bind to thymine over adenine, cytosine, and guanine. The authors proved visible fluorescence quenching by performing spectroscopic experiments.

Although amber molecular mechanics was applied by Rajendar et al., electron donating effects of methyl group on hydrogen bonding with thymine and on the whole electron structure have not been<smiles></smiles>

C

Molecule $\mathbf{C}$ (Lumiflavin): $\mathrm{R}_{2}=\mathrm{CH}_{3}$

Fig. 1. Schematic structure of studied molecules with atom notation. 
properly analyzed. In addition, only one dimer configuration was examined. Since three more dimer patterns are possible, we decided to present a thorough computational analysis of thymine binding ability to lumichrome (7,8-dimethyl-alloxazine), 1-methyl-lumichrome and lumiflavin (Fig. 1). Partial aims of this study are: (1) to calculate optimal geometries of electroneutral monomer molecules and four model van der Waals dimers within electronic ground and lowest excited states; (2) to compare hydrogen bond lengths and interaction energies of model dimers; (3) to evaluate optical transitions contributing to the lowest energy transition in absorption and fluorescence spectra. These changes in photo-physical properties with respect to the non-interacting subsystems are discussed below.

\section{Computational details}

The Gaussian 16 program package was used to perform quantum chemical calculations (Frisch et al., 2016). Optimal geometries of the studied molecules were calculated by the Density Functional Theory (DFT) with the B3LYP hybrid functional (Lee et al., 1988; Becke, 1998) without any constraints (energy cut-off of $10^{-5} \mathrm{~kJ} \mathrm{~mol}^{-1}$, final RMS energy gradient under $\left.0.01 \mathrm{~kJ} \mathrm{~mol}^{-1} \mathrm{~A}^{-1}\right)$. The $6-31+\mathrm{G}^{* *}$ basis set of atomic orbitals was applied (Hariharan and Pople, 1973; Rassolov et al., 1998). Based on the B3LYP geometries, the vertical singlet transition energies and oscillator strengths between the initial and final electronic states were computed by time-dependent TD-DFT (Furche and Ahlrichs, 2002). The TD-DFT was also used to calculate the lowest excited state geometries and de-excitation energies corresponding to the fluorescence energies. True minima on the potential energy surface were confirmed by frequencies' inspection (no imaginary frequencies).

The molecules and molecular orbitals were visualized using the Molekel (Flukiger et al., 2002) and Avogadro (Hanwell et al., 2012) program packages. Dimer interaction energies of the investigated molecule with thymine supersystem were calculated within the supermolecular approach, including the empirical dispersion corrections. The Grimme's dispersion correction with Becke-Johnson damping function (GD3BJ) (Grimme et al., 2011) was used. The Basis Set Superposition Error (BSSE) (Boys and Bernardi, 1970) was corrected using the counterpoise method (Simon et al., 1996; Xantheas, 1996).

\section{Results and Discussion}

Optimal gas-phase geometries of the studied molecules are planar like in crystal structures of their derivatives 3-methyl-lumichrome or flavinium nitrate (Norrestam and Stensland, 1972; Wouters et al., 1995). However, compared to the symmetric benzene molecule in electronic ground state $\left(S_{0}\right)$, their aromaticity is slightly perturbed. The gasphase B3LYP/6-31+G** optimal bond length in benzene is $1.398 \AA$. Consecutive alternation of single and double bonds can be seen for the dimethylsubstituted ring shown in Fig. 2. For the electronic ground state $\left(\mathrm{S}_{0}\right)$, differences between these bonds changed from 0.03 to $0.06 \AA$. The longest carboncarbon interatomic distances were found for the $\mathbf{A}$ molecule while the shortest bonds occur in the $\mathbf{C}$ molecule. The middle ring containing $\mathrm{N}(5)$ and $\mathrm{N}(10)$ atoms in $\mathbf{A}$ and $\mathbf{B}$ molecules exhibits larger differences between the single and double carboncarbon bonds, i.e. 0.05 to $0.09 \AA$. For reference, in symmetric pyrazine gas-phase B3LYP/6-31+G** molecule, the $\mathrm{C}-\mathrm{N}$ distance is $1.340 \AA$ and $\mathrm{C}-\mathrm{C}$ distance is $1.398 \AA$. Although only one ring is aromatic, effective global $\pi$-conjugation is theoretically predicted. The shortest distance of $1.299 \AA$ was found for the $\mathrm{C}(4 \mathrm{a})-\mathrm{N}(5)$ bond. Electronic excitation to the $S_{1}$ state can change the investigated bond lengths significantly. Maximal changes were found between the carbon and nitrogen atoms (see Fig. 2). Within supramolecular chemistry, the presence of a guest (the analyte) at the host site (the sensor) in chemosensing results in recognition signal (sensing) which can be monitored in real time. This requires the binding of the analyte to the receptor using all kinds of binding interactions such as hydrogen bonding, dipole- and electrostatic interactions, metal chelation, etc.

Potential of lumichrome derivatives as bioorganic molecular sensors was tested by examination of van der Waals-bounded (vdW) supersystems with thymine. As it was reported by Rajendar et al. (2010), methyl-substitution and benzo-annellation of lumazine to lumichrome or its derivatives increase the binding affinity significantly. Compared to alloxazine with the binding constants of $0.7 \times 10^{6} \mathrm{M}^{-1}$ obtained from fluorescence titration analysis, lumichrome and lumiflavin bind to thymine with remarkably higher affinity of $16 \times 10^{6} \mathrm{M}^{-1}$ and $21 \times 10^{6} \mathrm{M}^{-1}$, respectively (Sankaran et al., 2009). Moreover, the methyl group of thymine seems to increase the molecular polarizability which allows more favorable van der Waals interaction determined by the coulombic and dispersion forces. Four different dimer configurations with two intermolecular $\mathrm{O} \cdot \mathrm{H}$ hydrogen bonds are possible (Fig. 3). These can be explained by local dipolar pairs of atoms and not by global molecular static dipole moments. The supermolecular electronic interaction energies collected in Tab. 1 confirmed the thermodynamic stabil- 


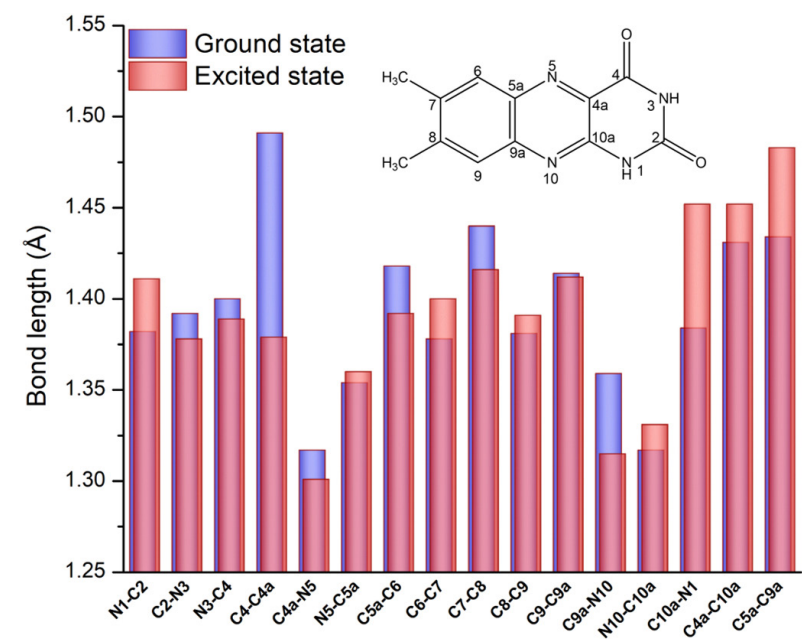

A

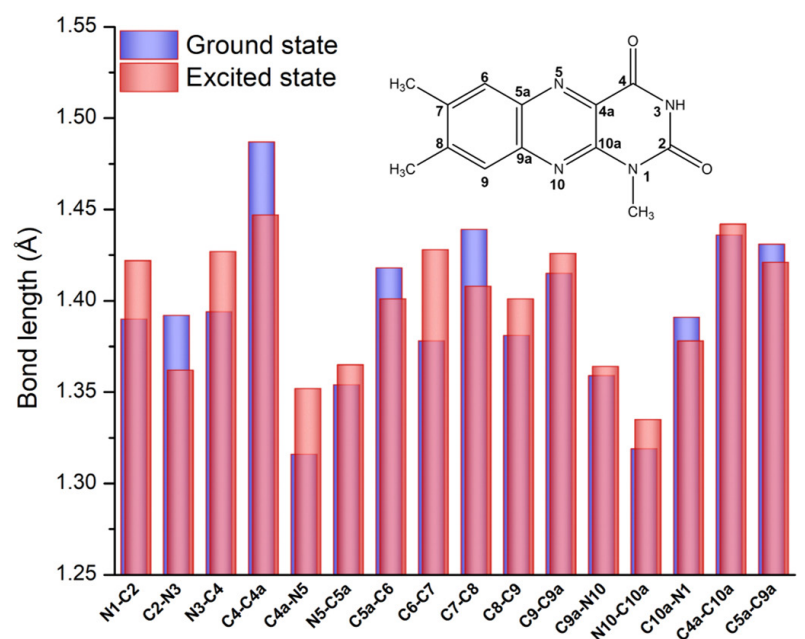

B

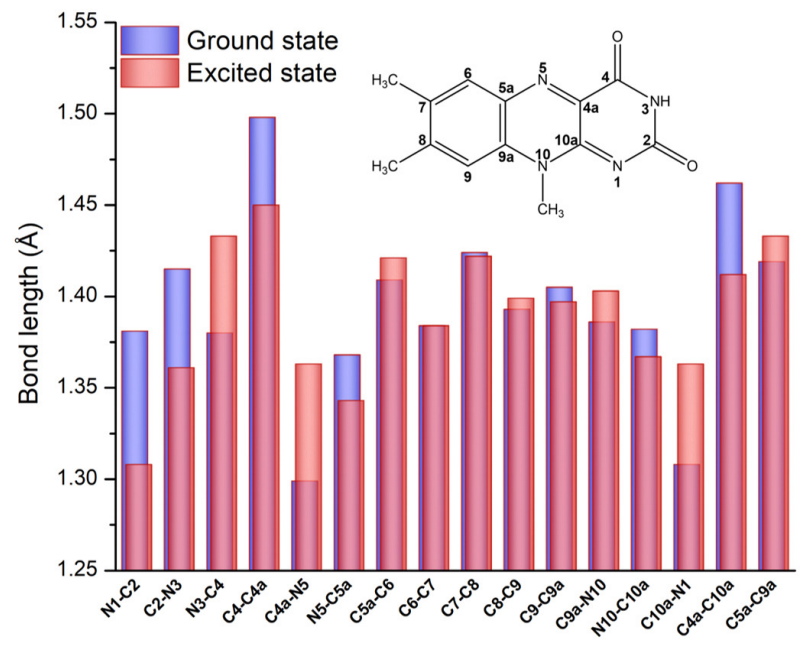

C

Fig. 2. B3LYP/6-31+G** optimal bond lengths (in Angströms) of electronic ground state $\left(\mathrm{S}_{0}\right)$ and the lowest excited state $\left(\mathrm{S}_{1}\right)$ in $\mathrm{A}, \mathrm{B}$ and $\mathrm{C}$ molecules.

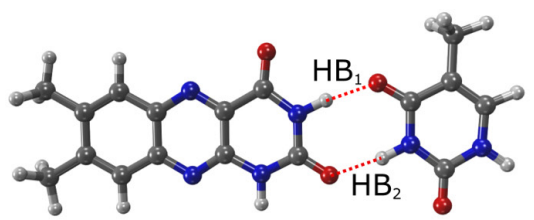

D1

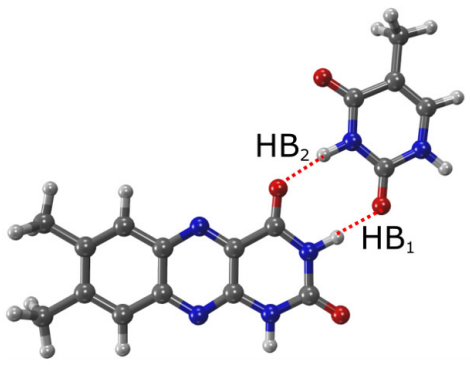

D3

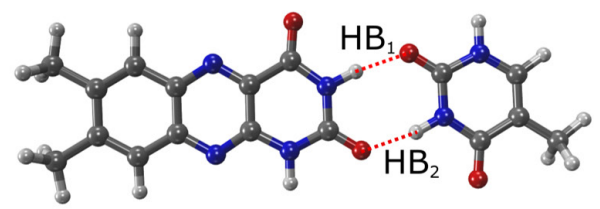

D2

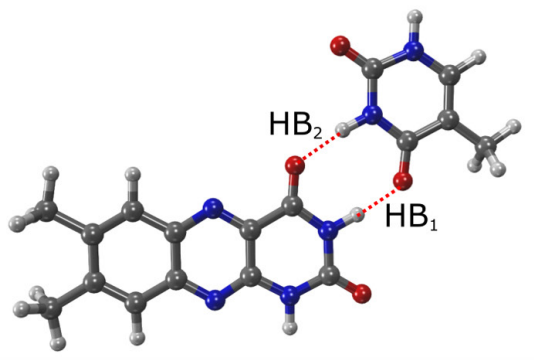

D4

Fig. 3. Notation of relative arrangement of studied dimers and notation of hydrogen $\mathrm{HB}_{1}$ and $\mathrm{HB}_{2}$ bonds. 
ity of all arrangements, with the longitudinal planar one (D1) being the most stable. This agrees with the recent force-field simulations of lumichrome interaction with thymine (Rajendar et al., 2010). However, energy differences between different arrangements can be considered negligible, therefore, occurrence of all the arrangements in a real sample is predicted according to the Boltzmann distribution.

When correction on BSSE is included in full geometry optimization, the interaction energy is slightly increased by up to $1.8 \mathrm{~kJ} \mathrm{~mol}^{-1}$. Otherwise, the trends in evaluated interaction energies are comparable (Tab. 1). Dimer stability is also directly influenced by hydrogen bond lengths (see notation in Fig. 3). The length of intermolecular hydrogen bond $\mathrm{HB}_{1}$ increases concurrently with the decreasing $\mathrm{HB}_{2}$ length (Tab. 2). It is obvious that shorter hydrogen bond lengths stabilize the supersystem resulting in a more negative interaction energy. The most stable dimer configuration is D1 with optimal methyl group orientation while D4 is slightly less stable. In case of lumiflavin, D2 is energetically more favorable than $\mathrm{D} 4$.

The application of chemosensors is a form of molecular recognition. Usually, the change (signal) during chemosensing is observed by measuring various physical properties of the chemosensor, such as the photophysical properties demonstrated in absorption or emission. Signaling is often optically based on electromagnetic radiation which causes changes in either (or both) the ultraviolet and visible absorption or the emission properties of the sensors. Moreover, electronic structure and its connection with the chemical modification can be described in terms of vertical transitions. Comparison of the energies for singlet states of the studied non-interacting monomers and model dimers is listed in Tab. 3.

Lumichrome (A) energy of the first excited vertical singlet state $\left(\mathrm{S}_{1}\right)$ over the electronic ground state is $3.39 \mathrm{eV}(366 \mathrm{~nm})$ and it is assigned to the HOMO to LUMO transition. However, B3LYP also predicts the third (HOMO- $\rightarrow$ LUMO) transition with significant absorption probability (86\%) and the oscillator strength of 0.22 , which corresponds with experimental optical absorption at $3.50 \mathrm{eV}$ (354 nm) (Sheraz et al., 2014; Maity et al., 2015, Sikorski et al., 2001). Slightly increased aromaticity of the keto-groups bearing ring in molecules $\mathbf{B}$ and $\mathbf{C}$ probably leads to the bathochromic shifts of the lowest energy transitions. Excitation energy of the $S_{0} \rightarrow S_{1}$ optical transition is $3.00 \mathrm{eV}(413 \mathrm{~nm})$

Tab. 1. Supermolecular interaction energies of vdW dimers in $\mathrm{S}_{0}$ state obtained for fully optimized geometries $(I E)$ and geometries optimized with correction on BSSE $\left(I E_{\mathrm{BSSE}}\right)$. Values are in $\mathrm{kJ} \mathrm{mol}^{-1}$.

\begin{tabular}{|c|c|c|c|c|c|c|c|c|}
\hline \multirow{2}{*}{$\begin{array}{c}\text { Dimers } \\
\text { Molecule }\end{array}$} & \multicolumn{2}{|c|}{ D1 } & \multicolumn{2}{|c|}{ D2 } & \multicolumn{2}{|c|}{ D3 } & \multicolumn{2}{|c|}{ D4 } \\
\hline & $I E$ & $I E_{\mathrm{BSSE}}$ & $I E$ & $I E_{\mathrm{BSSE}}$ & $I E$ & $I E_{\mathrm{BSSE}}$ & $I E$ & $\boldsymbol{I} \boldsymbol{E}_{\mathrm{BSSE}}$ \\
\hline $\mathbf{A}$ & -59.0 & -60.5 & -57.1 & -58.3 & -56.9 & -58.2 & -58.7 & -60.5 \\
\hline B & -58.5 & -59.6 & -56.7 & -57.8 & -56.2 & -57.7 & -58.2 & -60.0 \\
\hline C & -58.1 & -60.0 & -56.2 & -57.8 & -53.0 & -53.9 & -54.5 & -55.7 \\
\hline
\end{tabular}

Tab. 2. Hydrogen bond lengths $\mathrm{HB}_{1}$ and $\mathrm{HB}_{2}$ (in $\AA$ ) of vdW dimers in $\mathrm{S}_{0}$ state obtained for fully optimized geometries and geometries optimized with correction on BSSE. Values in parentheses stand for the hydrogen bond lengths in optimized $S_{1}$ state of dimers.

\begin{tabular}{|c|c|c|c|c|}
\hline \multirow{2}{*}{$\begin{array}{c}\text { System } \\
\text { Molecule-Dimer }\end{array}$} & \multicolumn{2}{|c|}{ without correction } & \multicolumn{2}{|c|}{ BSSE correction } \\
\hline & HB 1 & HB2 & HB 1 & HB2 \\
\hline A-D1 & $1.788(1.802)$ & $1.827(1.840)$ & 1.800 & 1.841 \\
\hline A-D2 & $1.804(1.785)$ & $1.842(1.826)$ & 1.817 & 1.856 \\
\hline A-D3 & $1.795(1.788)$ & $1.848(1.761)$ & 1.808 & 1.862 \\
\hline A-D4 & $1.778(1.806)$ & $1.833(1.775)$ & 1.790 & 1.847 \\
\hline B-D1 & $1.798(1.841)$ & $1.813(1.823)$ & 1.810 & 1.826 \\
\hline B-D2 & $1.814(1.836)$ & $1.827(1.857)$ & 1.828 & 1.841 \\
\hline B-D3 & $1.804(1.805)$ & $1.840(1.769)$ & 1.817 & 1.853 \\
\hline B-D4 & $1.787(1.785)$ & $1.825(1.755)$ & 1.799 & 1.838 \\
\hline C-D1 & $1.815(1.833)$ & $1.787(1.816)$ & 1.827 & 1.798 \\
\hline C-D2 & $1.831(1.832)$ & $1.802(1.847)$ & 1.845 & 1.813 \\
\hline C-D3 & $1.846(1.752)$ & $1.830(1.824)$ & 1.862 & 1.843 \\
\hline C-D4 & $1.831(1.732)$ & $1.816(1.807)$ & 1.845 & 1.829 \\
\hline
\end{tabular}


Tab. 3. Selected TD-B3LYP/6-31+G** lowest energy singlet $\left(\mathrm{S}_{0} \rightarrow \mathrm{S}_{\mathrm{n}}\right)$ transition energies $E$ (in eV), wavelengths $\lambda$ (in $\mathrm{nm}$ ) and the corresponding oscillator strengths $f$.

\begin{tabular}{|c|c|c|c|c|c|c|c|c|c|c|c|c|c|c|}
\hline System & $n$ & $E$ & $\lambda$ & $f$ & System & $n$ & $E$ & $\lambda$ & $f$ & System & $n$ & $E$ & $\lambda$ & $f$ \\
\hline \multirow[t]{4}{*}{$\mathbf{A}$} & 1 & 3.39 & 366 & 0.06 & B & 1 & 3.36 & 369 & 0.20 & C & 1 & 2.99 & 415 & 0.20 \\
\hline & 2 & 3.44 & 360 & 0.00 & & 2 & 3.42 & 363 & 0.00 & & 2 & 3.14 & 395 & 0.00 \\
\hline & 3 & 3.86 & 322 & 0.22 & & 3 & 3.85 & 322 & 0.19 & & 3 & 3.31 & 375 & 0.00 \\
\hline & 4 & 3.97 & 312 & 0.00 & & 4 & 3.94 & 315 & 0.00 & & 4 & 3.79 & 327 & 0.15 \\
\hline \multirow[t]{2}{*}{ A-D1 } & 1 & 3.40 & 364 & 0.00 & B-D1 & 1 & 3.38 & 367 & 0.00 & C-D1 & 1 & 3.03 & 410 & 0.24 \\
\hline & 2 & 3.44 & 361 & 0.08 & & 2 & 3.41 & 363 & 0.10 & & 2 & 3.03 & 409 & 0.00 \\
\hline \multirow[t]{2}{*}{ A-D2 } & 1 & 3.41 & 364 & 0.00 & & 3 & 3.78 & 328 & 0.00 & & 3 & 3.07 & 404 & 0.00 \\
\hline & 2 & 3.43 & 361 & 0.08 & B-D2 & 1 & 3.38 & 366 & 0.00 & C-D2 & 1 & 3.03 & 410 & 0.24 \\
\hline \multirow[t]{2}{*}{ A-D3 } & 1 & 3.35 & 370 & 0.08 & & 2 & 3.41 & 364 & 0.10 & & 2 & 3.07 & 404 & 0.00 \\
\hline & 2 & 3.45 & 359 & 0.00 & & 3 & 3.80 & 327 & 0.00 & & 3 & 3.16 & 393 & 0.00 \\
\hline \multirow[t]{6}{*}{ A-D4 } & 1 & 3.35 & 370 & 0.08 & B-D3 & 1 & 3.32 & 374 & 0.10 & C-D3 & 1 & 2.95 & 420 & 0.22 \\
\hline & 2 & 3.45 & 360 & 0.00 & & 2 & 3.43 & 362 & 0.00 & & 2 & 3.17 & 391 & 0.00 \\
\hline & & & & & & 3 & 3.67 & 338 & 0.00 & & 3 & 3.18 & 390 & 0.00 \\
\hline & & & & & B-D4 & 1 & 3.27 & 379 & 0.10 & C-D4 & 1 & 2.95 & 421 & 0.22 \\
\hline & & & & & & 2 & 3.42 & 363 & 0.00 & & 2 & 3.15 & 393 & 0.00 \\
\hline & & & & & & 3 & 3.64 & 341 & 0.00 & & 3 & 3.16 & 392 & 0.00 \\
\hline
\end{tabular}

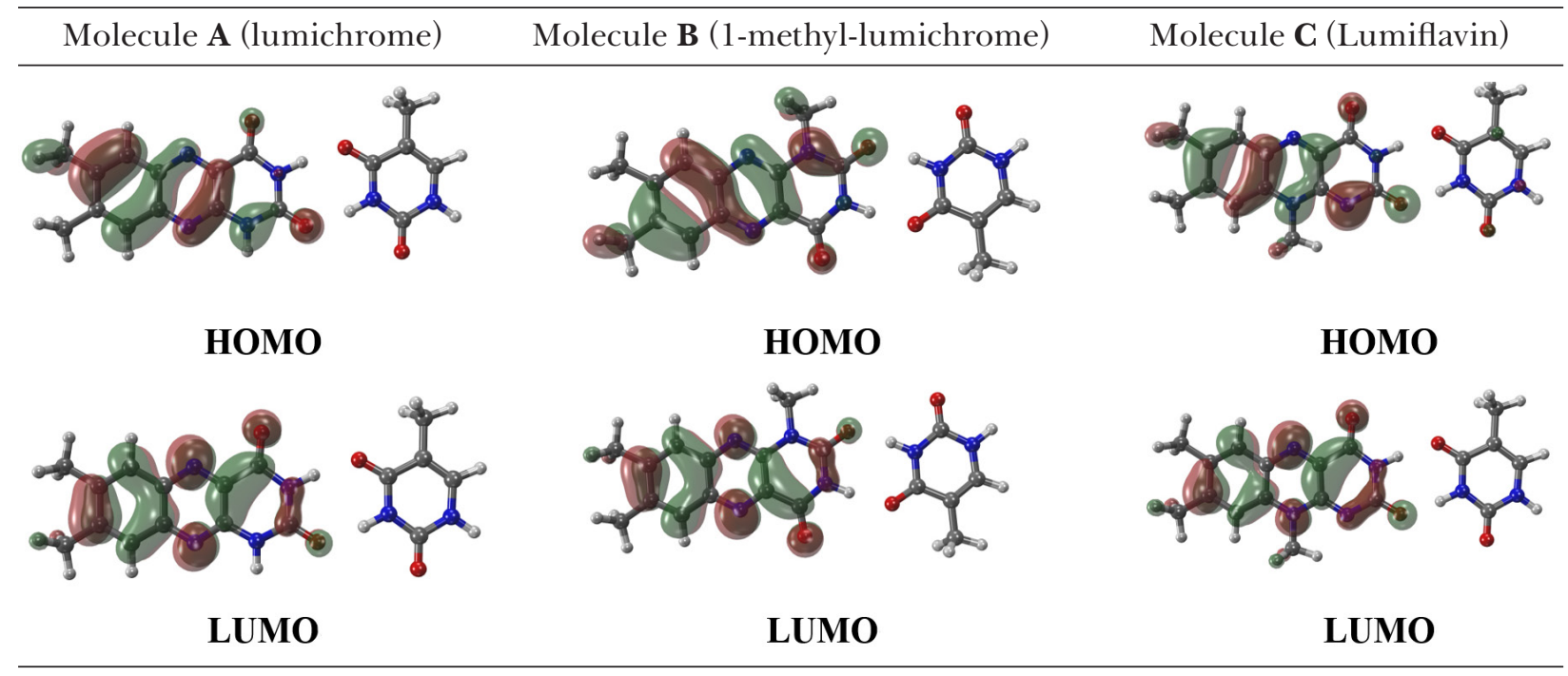

Fig. 4. Shapes of B3LYP/6-31+G** selected molecular orbitals of model D1 dimer for thymine and studied molecules. Depicted iso-surface value is 0.025 a.u.

and $2.99 \mathrm{eV}(415 \mathrm{~nm})$ for $\mathbf{B}$ and $\mathbf{C}$, respectively, indicating methyl groups attached to the $\mathrm{N}(1)$ or $\mathrm{N}(10)$ atoms to increase the first transition oscillator strength up to 0.20 (compare lumichrome $\mathbf{A}$ with $\mathbf{B}$ and $\mathbf{C})$. Experimental absorption bands at $368 \mathrm{~nm}$ and $441 \mathrm{~nm}$ for lumiflavin $\mathbf{C}$ in aqueous solution were measured by Sheraz et al. (2014). It seems that the corresponding theoretical values with significant oscillator strengths are $3.79 \mathrm{eV}(327 \mathrm{~nm})$ and $2.99 \mathrm{eV}(415 \mathrm{~nm})$, respectively.

Interestingly, bonding with thymine causes a negligible shift of vertical $\mathrm{S}_{0} \rightarrow \mathrm{S}_{1}$ transition energies. Red shift up to $0.04 \mathrm{eV}$ was found for $\mathrm{D} 1$ and
D2 supersystems and blue shift up to $0.09 \mathrm{eV}$ for the D3 and D4 ones. Also, in case of D1 and D2 dimer configurations containing $\mathbf{A}$ and $\mathbf{B}$ molecules, $\mathrm{HOMO} \rightarrow$ LUMO transition is the second $\mathrm{S}_{0} \rightarrow \mathrm{S}_{2}$ transition with higher oscillator strength while in other supersystems it corresponds to the first vertical excitation. The first vertical $S_{0} \rightarrow S_{1}$ transition in these four cases (A-D1/D2, B-D1/D2) match the HOMO-3 $\rightarrow$ LUMO transition.

Graphical frontier molecular orbitals analysis in Fig. 4 shows negligible contribution of the thymine molecule. HOMO electrons are uniformly delocalized over the longest axial direction. Similarity 
Tab. 4. Selected vertical TD-B3LYP/6-31+G** de-excitation $\left(\mathrm{S}_{1} \rightarrow \mathrm{S}_{0}\right)$ energies $E$ (in eV), wavelengths $\lambda$ (in $\mathrm{nm}$ ) and oscillator strengths $f$ estimated for the lowest excited singlet states.

\begin{tabular}{lcclcllll}
\hline System & $\boldsymbol{E}(\lambda)$ & $\boldsymbol{f}$ & System & $\boldsymbol{E}(\lambda)$ & $\boldsymbol{f}$ & System & $\boldsymbol{E}(\lambda)$ & $f$ \\
\hline A & $2.77(448)$ & 0.00 & B & $2.89(430)$ & 0.05 & C & $1.95(637)$ & 0.00 \\
A-D1 & $2.79(444)$ & 0.00 & B-D1 & $2.95(421)$ & 0.06 & C-D1 & $2.54(488)$ & 0.18 \\
A-D2 & $2.79(444)$ & 0.00 & B-D2 & $2.94(422)$ & 0.06 & C-D2 & $2.53(490)$ & 0.17 \\
A-D3 & $2.85(436)$ & 0.05 & B-D3 & $2.84(436)$ & 0.06 & C-D3 & $1.81(684)$ & 0.00 \\
A-D4 & $2.85(436)$ & 0.05 & B-D4 & $2.84(437)$ & 0.06 & C-D4 & $1.80(688)$ & 0.00 \\
\hline
\end{tabular}

between the shapes of the orbitals in dimers and non-interacting monomers suggests minimal effect on the chromophore confirming small differences in vertical transitions. The thymine molecule does not contribute with its own electrons to these excitations.

Moreover, transition from the $S_{0}$ state to the first excited state is related to the significant change in hydrogen bond lengths for the D1 dimer (Tab. 2). Rajendar et al. (2010) found fluorescence peak broadening after mixing lumichrome and 1-methyl-lumichrome with thymine. Indeed, the $S_{1} \rightarrow S_{0}$ de-excitation energies of D1 and D2 dimers show small blue shifts compared to the non-interacting molecules (Tab. 4). Therefore, pairing with thymine is expected to provide a visible change in the fluorescence spectra. The increase of fluorescence oscillator strength up to 0.17 is predicted for $\mathbf{C - D 1}$ and $\mathbf{C - D 2}$ dimers. Despite higher oscillator strength prediction, the theoretical TD-CAM-B3LYP de-excitation energies were not included because of significant energy overestimation. According to experimental data measured by Rajendar et al. (2010), fluorescence maximum of free-of-DNA lumichrome $\mathbf{A}$ is at $476 \mathrm{~nm}$ (2.60) with three-times higher fluorescence intensity than in the supersystem with thymine (A-D1). Position of the peak for the supersystem is negligibly shifted to $473 \mathrm{~nm}(2.62 \mathrm{eV})$. The shift agrees well with theoretical values in the present study; however, the TD-B3LYP method overestimates the de-excitation energies. In case of 1-methyl-lumichrome $\mathbf{B}$, maxima were observed at $474 \mathrm{~nm}$ and $473 \mathrm{~nm}(2.62 \mathrm{eV})$ for the free-of-DNA form and $\mathbf{B}$ binding to thymine, respectively. This also agrees well with the experimental value of $475 \mathrm{~nm}$ measured for both lumichrome $\mathbf{A}$ and 1-methyl-lumichrome $\mathbf{B}$ in aqueous solution by Sikorski et al. (2001). They have also shown that lumiflavin $\mathbf{C}$ exhibits fluorescence maximum at about $530 \mathrm{~nm}$.

\section{Conclusion}

Optimal geometries and electronic structure of lumichrome, 1-methyl-lumichrome and lumi- flavin in the electronic ground state and in the lowest excited states were theoretically studied. Compared with the reference lumichrome, the addition of electron donating methyl groups to the $\mathrm{N}(1)$ or $\mathrm{N}(10)$ position had a significant effect on the bond lengths in the benzo[g]pteridine backbone. The lowest predicted vertical excitation energy for lumichrome is $3.39 \mathrm{eV}$ whereas these energies for $\mathbf{B}$ and $\mathbf{C}$ molecules are red shifted. These vertical electronic excitation energies correspond to the HOMO to LUMO transition of the $\Pi$-type character. Two stable intermolecular hydrogen bonds with thymine were confirmed by the theoretical calculations of interaction energies of four model van der Waals complexes. For all the studied dimers, optical transitions with sufficient oscillator strengths corresponding to the $\mathrm{HOMO} \rightarrow$ LUMO transition are predicted. Only small blue shifts of de-excitation energies in the fluorescence spectra of the supersystems were found compared with the monomers. Even though the used quantum mechanical approaches have limitations, the obtained theoretical trends in the (de-)excitation energies correspond well with experimental observations. Thus, theoretical analysis could help in chemosensors modeling and identification of new organic candidates for chemosensing. The studied molecules can be used as chemosensors in DNA due to its high binding ability and formation of stable van der Waals dimers with thymine.

\section{Acknowledgement}

The work has been supported by Slovak Research and Development Agency (APVV-15-0053) and VEGA 1/0504/20. We are grateful to the HPC center at the Slovak University of Technology in Bratislava, which is a part of the Slovak Infrastructure of High Performance Computing (SIVVP project, ITMS code 26230120002, funded by the European region development funds, $E R D F)$ for the computational time and resources made available. D.C. would like to thank for support from the STU Grant scheme for Support of Young Researchers (1848). 


\section{References}

Becke AD (1998) Phys Rev A 38(6): 3098-3100. Boys SF, Bernardi F (1970) Mol. Phys. 19: 553-566.

Flukiger P, Luthi HP, Sortmann S, Weber J (2002) Molekel 4.3, Swiss National Supercomputing Centre, Manno, Switzerland.

Frisch MJ, Trucks GW, Schlegel HB, Scuseria GE, Robb MA, Cheeseman JR, Scalmani G, Barone V, Petersson GA, Nakatsuji H, Li X, Caricato M, Marenich AV, Bloino J, Janesko BG, Gomperts R, Mennucci B, Hratchian HP, Ortiz JV, Izmaylov AF, Sonnenberg JL, Williams-Young D, Ding F, Lipparini F, Egidi F, Goings J, Peng B, Petrone A, Henderson T, Ranasinghe D, Zakrzewski VG, Gao J, Rega N, Zheng G, Liang W, Hada M, Ehara M, Toyota K, Fukuda R, Hasegawa J, Ishida M, Nakajima T, Honda Y, Kitao O, Nakai H, Vreven T, Throssell K, Montgomery JA Jr., Peralta JE, Ogliaro F, Bearpark MJ, Heyd JJ, Brothers EN, Kudin KN, Staroverov VN, Keith TA, Kobayashi R, Normand J, Raghavachari K, Rendell AP, Burant JC, Iyengar SS, Tomasi J, Cossi M, Millam JM, Klene M, Adamo C, Cammi R, Ochterski JW, Martin RL, Morokuma K, Farkas O, Foresman JB, Fox DJ (2016) Gaussian 16, Revision B.01, Gaussian, Inc., Wallingford CT.

Furche F, Ahlrichs R (2002) J Chem Phys. 117(16): 7433.

Gao J, Liu H, Kool ET (2005) Angew. Chem. Int. Ed. 44: 3118-3122.

Grimme S, Ehrlich S, Goerigk L (2011) J. Comput. Chem. 32: 1456-1465.

Hagihara S, Kumasawa H, Goto Y, Hayashi G, Kobori A, Saito I, Nakatani K (2004) Nucleic Acids Res. 32: 278-286.

Hanwell MD, Curtis DE, Lonie DC, Vandermeersch T, Zurek E, Hutchison GR (2012) J Cheminform. 4(1): 17.

Hariharan PC, Pople JA (1973) Theor. Chim. Acta. 28(3): 213-222.
Kim TW, Kool ET (2005) J. Org. Chem. 70: 2048-2053.

Lee AH, Kool ET (2005) J. Am. Chem. Soc. 127: 3332-3338.

Lee C, Yang W, Parr RG (1988) Phys Rev B. 37(2): $785-789$.

Maity B, Chatterjee A, Seth D (2015) RSC Adv. 5: 3814-3824.

Norrestam R, Stensland B (1972) Acta Crystallographica B: Struct. Crystallogr. Cryst. Chem., 28: 440.

Raibenspies JH, Guo F, Rizzo CJ (2000) Org. Lett. 2(7): 903-906.

Rajendar B, Rajendran A, Ye Z, Kanai E, Sato Y, Nishizawa S, Sikorski M, Teramae N (2010) Org. Biomol. Chem. 8: 4949-4959.

Rajendran A, Nair BU (2006) Biochim. Biophys. Acta 1760: 1794-1801.

Rassolov VA, Pople JA, Ratner MA, Windus TL (1998) J Chem Phys. 109(4): 1223-1229.

Sankaran NB, Sato Y, Sato F, Rajendar B, Morita K, Seino T, Nishizawa S, Teramae N (2009) J. Phys. Chem. B 113: 1522-1529.

Sheraz MA, Kazi SH, Ahmed S, Qadeer K, Khan MF, Ahmad I (2014) Cent. Eur. J. Chem. 12(6): 635-642.

Sikorski M, Sikorska E, Koziolowa A, Gonzalez Moreno R, Bourdelande JL, Steer RP, Wilkinson F (2001) J. Photochem. Photobiol. B 60: 114-119.

Simon S, Duran M, Dannenberg JJ (1996) J. Chem. Phys. 105: 11024-11031.

White S, Szewczyk JW, Turner JM, Baird EE, Dervan PB (1998) Nature 391: 468-471.

Wouters J, Evrard G, Durant F (1995) Acta Cryst. C51: 1223-1227.

Wu D, Sedgwick AC, Gunnlaugsson T, Akkaya EU, Yoon J, James TD (2017) Chem. Soc. Rev. 46(23): 7105-7123. Xantheas SS (1996) J. Chem. Phys. 104: 8821-882. 